\title{
Single- and double-beam reflectarrays for Ka band communication
}

\author{
RANIA ELSHARKAWY ${ }^{1, *}$, MOATAZA HINDY $^{1}$, A -R SEBAK ${ }^{2}$, ADEL SALEEB $^{3}$, \\ EL-SAYED M EL-RABAIE ${ }^{3}$, AMR RAGHEB $^{4}$, MUHAMMAD ASHRAF $^{4}$ and \\ SALEH ALSHEBEILI ${ }^{4,5}$ \\ ${ }^{1}$ Electronics Research Institute, Dokki, Giza, Egypt \\ ${ }^{2}$ Department of Electrical and Computer Engineering, Concordia University Montreal, Montreal, QC, Canada \\ ${ }^{3}$ Faculty of Electronic Engineering, Menoufia University, Menouf 32952, Egypt \\ ${ }^{4}$ KACST-TIC in Radio Frequency and Photonics for the e-Society (RFTONICS), King Saud University, \\ Riyadh 11421, Saudi Arabia \\ ${ }^{5}$ Department of Electrical Engineering, King Saud University, Riyadh 11421, Saudi Arabia \\ e-mail: raniarefaat85@eri.sci.eg; m1_hendi@yahoo.com; abdo@ece.concordia.ca; adel48saleeb@yahoo.com; \\ srabie1@yahoo.com; aragheb@ksu.edu.sa; mashraf@ksu.edu.sa; dsaeh@ksu.edu.sa
}

MS received 13 October 2017; revised 8 August 2018; accepted 31 October 2018; published online 6 April 2019

\begin{abstract}
The Ka band has found applications in satellite, and radar communications. It is also expected that this band will be utilized for $5 \mathrm{G}$ applications. This paper presents single- and double-beam microstrip reflectarrays with single layer and compact size for Ka band communications at $28 \mathrm{GHz}$. Three different unit cells are investigated in this paper. Single- and double-beam reflectarrays are investigated. The reflectarrays are designed at $28 \mathrm{GHz}$ with a physical size of $10 \lambda \times 10 \lambda$. A pyramidal horn antenna is used for the feeding purpose. The focal-length-to-diameter (F/D) ratio is equal to one. Two different scenarios for single-beam reflectarrays are presented: one with a broadside direction and the other with a $10^{\circ}$ tilt angle. The simulation results show that for the broadside single-beam scenario, it is possible to achieve a gain up to $28.5 \mathrm{~dB}$, and a 1-dB gain-bandwidth up to $30.7 \%$. On the other hand, the presented reflectarray for the single-beam design at $10^{\circ}$ tilt angle gives a gain of about $26.4 \mathrm{~dB}$, a side lobe level (SLL) of about $-15.6 \mathrm{~dB}$, and a $19.3 \%$ gain-bandwidth. For the double-beam reflectarray, four different designs at different angles of $5^{\circ}, 10^{\circ}, 15^{\circ}$, and $20^{\circ}$ have been simulated and compared. Moreover, the simulation results on the double-beam reflectarray show that the double-beam design at $10^{\circ}$ is better from the gain and SLL perspectives. Two prototypes for broadside single-beam reflectarrays have been fabricated and measured. The measurement results show a good match with the simulation results. Gain flatness is guaranteed for both the simulated and measured results over the band of interest.
\end{abstract}

Keywords. Ka band; reflectarray; 5G; single-beam reflectarray; double-beam reflectarray.

\section{Introduction}

In future 5G wireless communication systems, large bandwidths are required. To achieve this objective, there is a need to work at high frequencies [1]. This, in turn, makes the Ka band a good candidate for future-generation wireless communication systems. Operation at the Ka band has several advantages such as the large bandwidth and consequently the large capacity, and the reduction of the antenna sizes due to the small wavelengths [1]. The main problem in this band is the high path loss and the attenuation due to the rain and atmospheric absorption $[1,2]$. In this paper, reflectarray antennas are designed at $28 \mathrm{GHz}$, because the level of attenuation due to atmospheric absorption at this frequency is not too high. It is equal to

*For correspondence about $0.7 \mathrm{~dB}$ per $100 \mathrm{~m} \mathrm{[1].} \mathrm{In} \mathrm{future} 5 \mathrm{G}$ communication systems, it is required to divide cells into micro- and picocells to increase the capacity through frequency reuse. Omni-directional antennas will no longer be suitable, because they increase interference [1]. Therefore, antennas, which can give pencil beams with high gain to minimize interference and at the same time maximize the gain, will be needed.

Two of the most common antennas that produce pencil beams and high gains are the parabolic reflectors and the phased-array antennas. A parabolic reflector depends on a primary feed horn to illuminate a metallic reflecting surface, which is designed by a method making all the path lengths from the focal point to any point on the parabolic surface equal in order to guarantee a unified direction for the reradiated field. The main disadvantages of the parabolic reflector is its huge size and large mass, especially at 


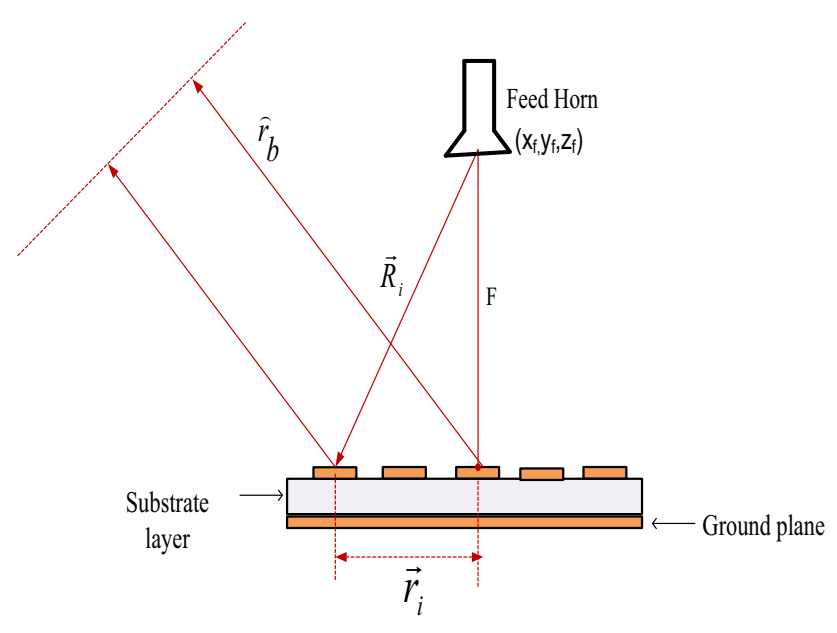

Figure 1. Reflectarray antenna.

high frequencies in the millimeter wave range. Moreover, parabolic reflectors exhibit high cross polarization levels [3]. In addition, phased-array antennas are composed of discrete elements and beamforming networks to produce the desired beam. The main disadvantage of the phasedarray antennas is the power loss in the feeding network as it uses phase shifters to adjust the phases of the elements. When they are equipped with controllable phase shifters, they become very expensive due to the complicated beamformers and the use of several high-cost amplifier modules. Although phased-array antennas are light-weight, low-profile antennas with low cross polarization levels, they have small bandwidths and large losses at microwave and millimeter wave frequencies [3].

Reflectarrays have the merits of both parabolic reflectors and phased arrays with compact size and efficient simple feeding [4]. Unlike the parabolic reflector, a classical reflectarray consists of a flat dielectric substrate material sandwiched between two conducting materials: the ground plane at the bottom surface and the conducting array patches on the top surface as shown in figure 1 [5]. Similar to the parabolic reflector, a reflectarray is fed by a horn antenna located at the focal point. The reflectarrays eliminate the complicated and expensive beamforming networks existing in phased-array antennas [4].

Reflectarray antennas can be designed to produce single or multiple beams using single or multiple feeds at different frequency bands [6-13]. Fang et al introduced a doublebeam reflectarray using a single feed horn based on the phase synthesis method, and achieved a gain of about $19.5 \mathrm{~dB}$ and a $1-\mathrm{dB}$ gain-bandwidth of $16.5 \%$ for the frequency range from 5 to $7 \mathrm{GHz}$ [8]. Arrebola et al introduced a three-beam reflectarray using three feed horns [9]. Their antenna is designed to operate at a center frequency of $25.5 \mathrm{GHz}$ for a Local Multi-point Distribution System (LMDS). It consists of two layers with $30 \times 30$ elements in each layer. The maximum obtained gain was around $20 \mathrm{~dB}$ and the gain-bandwidth achieved was $8 \%$.

Several researchers have investigated the design of reflectarray antennas to work at the Ka band. Abdelhady et al presented a single-beam Ka-band double-layer reflectarray antenna and achieved a gain of about $25 \mathrm{~dB}$ and a 3-dB-gain bandwidth of about 24\% [14]. Nayeri et al introduced a quad-beam reflectarray using the alternating projection method to optimize the phase distribution on the reflectarray surface $[6,7]$. They designed the reflectarray in the Ka band with a single feed. This array achieved a gain of about $27 \mathrm{~dB}$. Zhang presented a single-beam dielectric resonator reflectarray that works at $30 \mathrm{GHz}$ [15]. This array is three-dimensional in nature with dimensions of $120 \times 120 \mathrm{~mm}^{2}$. It achieved a gain of $28 \mathrm{dBi}$ at $30 \mathrm{GHz}$. An et al investigated the integration between reflectarray antennas working in the Ka band and solar cells [16].

As the $\mathrm{Ka}$ band is a good candidate for $5 \mathrm{G}$ communications, design of reflectarrays for $5 \mathrm{G}$ applications in $\mathrm{Ka}$ band has captured the interest of researchers $[17,18]$. Hong et al presented some suggestions for reflectarrays in 5G applications [17]. They recommended that multiple beams can be obtained from reflectarrays through the use of multiple horns to feed a single reflectarray. By increasing the number of horns, the number of beams is increased. This scenario of multiple horns may be cost inefficient. Hence, there is a need to generate multiple beams with a single horn. Dahri et al presented a review of the utilization of reflectarrays in 5G applications [18]. Several unit cells have been presented and investigated in this study for single-beam reflectarrays with the main target of enhancing the bandwidth at the cost of gain reduction.

Chaharmir et al in $[19,20]$ concluded that the linear phase versus element size is the basis of the broadband performance of a medium-size reflectarray. So, the current paper investigates this issue through the use of the superposition concept. In this paper, three different broadband unit cells are suggested for the design of the Ka band reflectarrays. The main objective is to use a unit cell with high bandwidth in order to enhance the overall reflectarray bandwidth. Based on the selected unit cells, reflectarrays with a single beam at broadside direction and another one with a beam at $10^{\circ}$ tilt angle will be introduced. In addition, double-beam reflectarrays generating beams at different angles using a single feed horn will be introduced and compared.

\section{Reflectarray theory}

The reflectarray shown in figure (1) is composed of an array of patches illuminated with a feed horn located at $\left(x_{f}\right.$, $\left.y_{f}, z_{f}\right)$. The total phase delay from the feed to a fixed aperture in front of the reflectarray must be constant for all elements. To collimate a beam in a certain direction with 
(a)

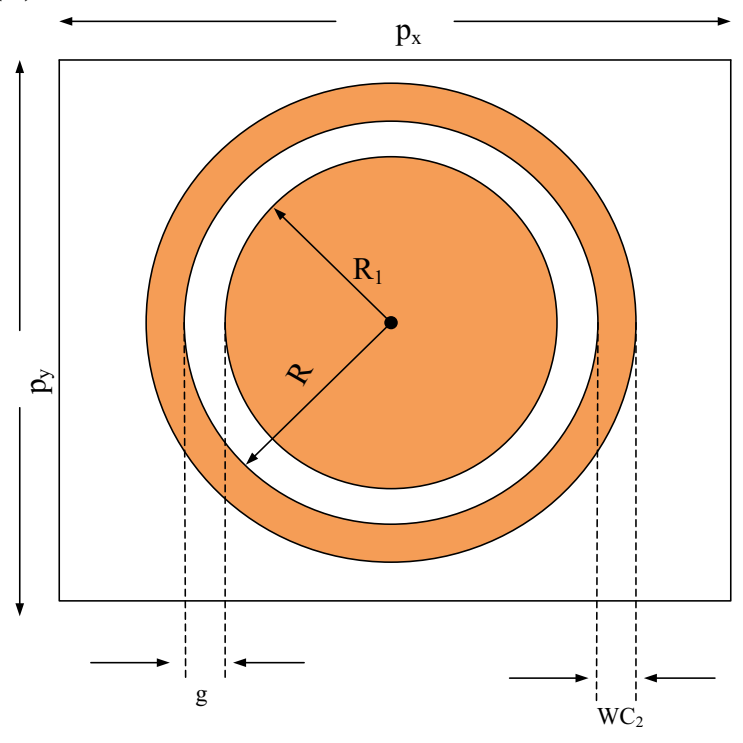

(c)

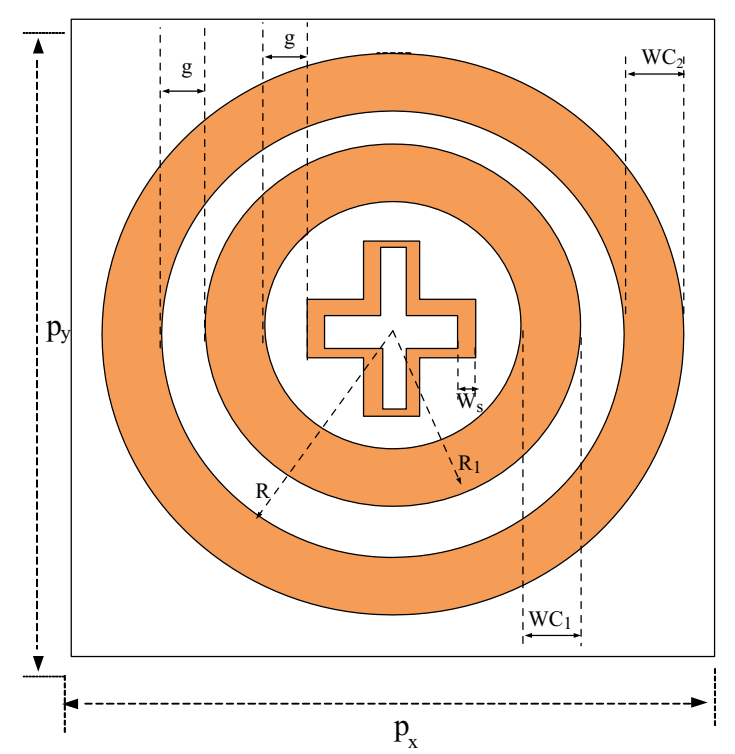

(b)

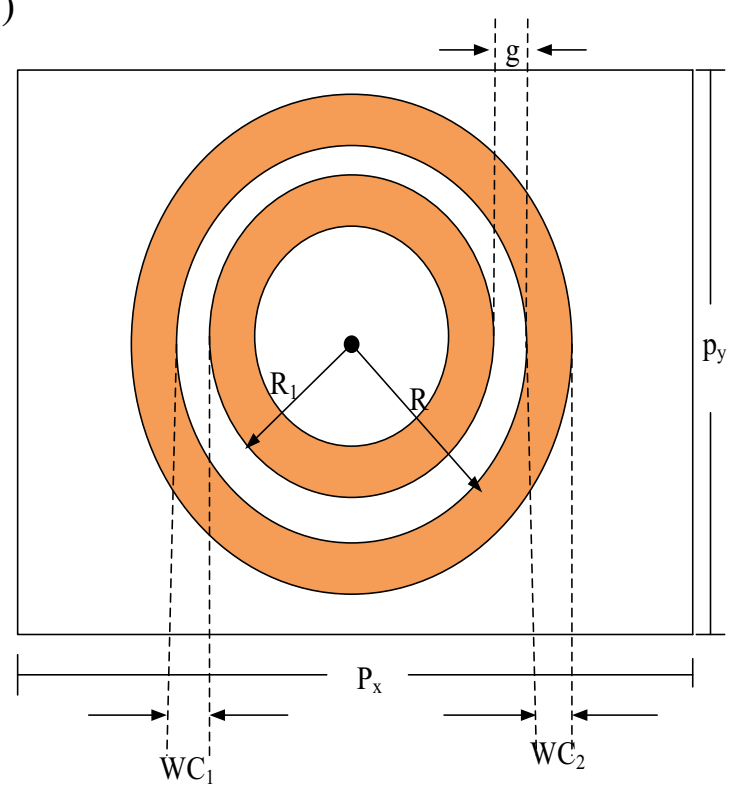

(d)
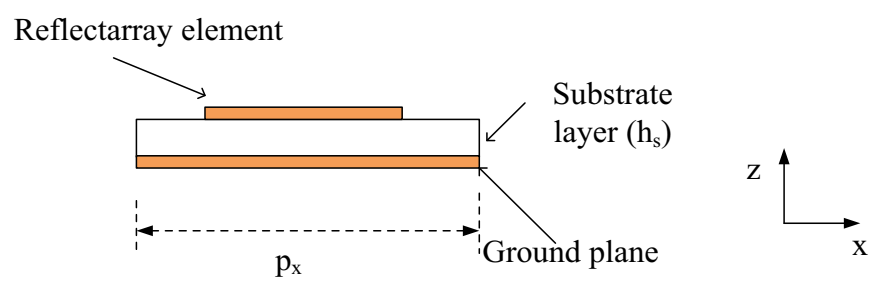

Figure 2. $\mathrm{UC}_{\mathrm{s}}$ configuration (a) $\mathrm{UC}_{1}$, (b) $\mathrm{UC}_{2}$, (c) $\mathrm{UC}_{3}$, (d) Side view. The dimensions of the $\mathrm{UC}$ are all in $\mathrm{mm} . P x=P y=10.7 / 2$, $R_{1}=R-\mathrm{g}, W c_{1}=0.2, W c_{2}=0.2, W s=0.2, \mathrm{~g}=0.214, h s=1.5748$, and $\varepsilon r=2.2$ (Rogers RT 5880).

$\left(\theta_{\mathrm{o}}, \phi_{\mathrm{o}}\right)$ considering that the reflection phase from each reflectarray element is dependent on its position, a compensation phase $\gamma_{i}$ is required to satisfy the following equation $[3,4]$ :

$$
k_{o}\left(R_{i}-\overrightarrow{r_{i}} \cdot \hat{r}_{b}\right)-\gamma_{i}=2 N \pi
$$

where $k_{o}=2 \pi / \lambda_{o}$ is the free-space wave number, $i$ is the element index, $N$ is an integer, and

$$
R_{i}=\sqrt{\left(x_{i}-x_{f}\right)^{2}+\left(y_{i}-y_{f}\right)^{2}+z_{f}^{2}}
$$

is the distance from the phase center of the feed horn to the center of the $i$ th reflectarray element, $\overrightarrow{r_{i}}$ is the position vector to the center of the reflectarray element from the reflectarray surface center, $\hat{r}_{b}$ is the unit vector in the collimated beam direction, $N$ is an integer, and $\gamma_{i}$ is the $i$ th element phase required to compensate for the delay resulting from the corresponding path. To satisfy Eq. (1) for 


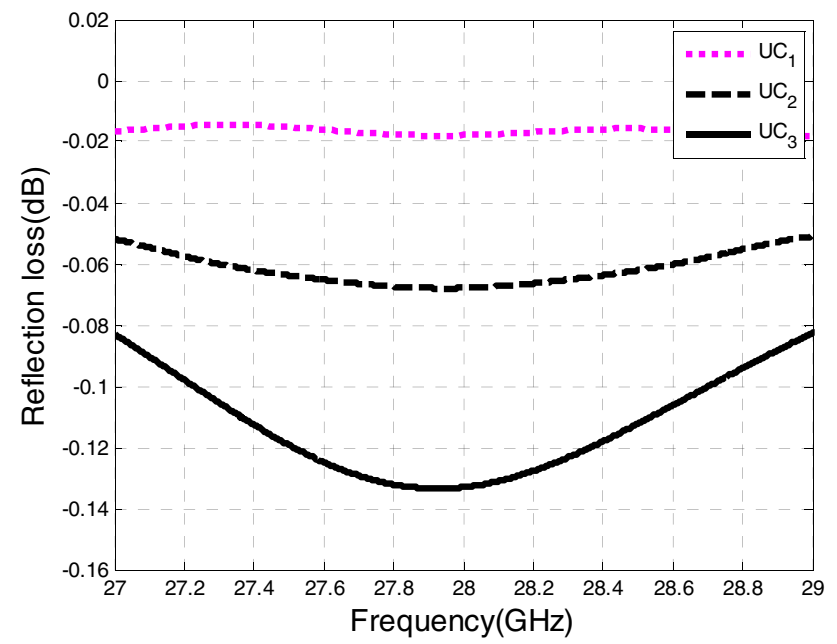

Figure 3. Reflection loss versus frequency for $\mathrm{UC}_{1}, \mathrm{UC}_{2}$, and $\mathrm{UC}_{3}$.

each element in the reflectarray, there is a single degree of freedom represented in the value of $\gamma_{i}$, which is varied by varying the element area as will be explained in sections 3 and 4.

\section{Structure of the unit cells}

In this paper, three different unit cells are presented as shown in figure 2 . The first one $\left(\mathrm{UC}_{1}\right)$ consists of a concentric ring with a solid circular patch. The second one $\left(\mathrm{UC}_{2}\right)$ consists of two concentric rings. The third one $\left(\mathrm{UC}_{3}\right)$ consists of two concentric rings with a solid cross loop inside. The dimensions of these unit cells are shown in figure 2 , where $p_{x}=p_{y}=\lambda / 2=5.35 \mathrm{~mm}$. The unit cells are designed to work around $28 \mathrm{GHz}$. The advantage of using a mixture of different elements in the unit cell is to increase its bandwidth. Consequently, the overall bandwidth of the reflectarray is increased. As each element resonates at a different frequency, this results in different transition regions in the phase-length characteristics of the unit cell [21-24]. The reflection loss curves versus frequency for the suggested unit cells are shown in figure 3. To build the reflectarrays, there is a need to satisfy Eq. (1) at each element of the reflectarray. This means that there is a need to change the dimensions of the unit cell inside the area $p_{x} \times p_{y}$ for each element in the reflectarray in order to achieve the required phase shift.

\section{Estimation of the reflection phase curve}

A reflection phase curve is estimated to relate the element size to its corresponding phase. This step is performed prior to the design of the reflectarray. The idea of estimating the reflection phase curve is based on assuming that a plane wave is incident on the unit cell and then reflected with a different amplitude due to attenuation and a different phase due to the unit cell size. The phase of the reflected wave is estimated as the reflection phase. This process is repeated for different sizes to produce a lookup table, which is used to plot the reflection phase curve. In this procedure, the infinite array approach is adopted. This approach assumes that for each size of the element, all surrounding elements are identical in size. The main advantage of this approach is that it takes into consideration the mutual coupling between elements.

For the proposed unit cells, the reflection phase curve is computed by varying the radius $\mathrm{R}$ shown in figure 4 . The CST simulator has been used for this purpose [25]. The reflection phase curve can be calculated using a Floquet port with periodic boundary conditions [4]. By this method, the reflection phase curve can be calculated at different incidence angles for the incident plane wave. Hence, the reflection phase curves can be adopted for different incidence angles. Another possible method for the estimation of the reflection phase curves is to use a parallel-plate waveguide port, where the two plates normal to the electric field direction are electric walls and the other two plates are magnetic walls [4]. The parallel-plate waveguide port enables the calculation of the reflection phase curve for normal incidence. Two main characteristics should be satisfied by the reflection phase curves: linearity to minimize the phase errors in the designed array, and a $360^{\circ}$ span at least. Figure 4 shows the reflection phase curves for the proposed unit cells with normal incidence at three different frequencies: $27 \mathrm{GHz}$, $28 \mathrm{GHz}$, and $29 \mathrm{GHz}$.

\section{Single-beam reflectarray}

Three reflectarrays have been designed based on the three proposed unit cells. All reflectarrays have dimensions of $10 \lambda \times 10 \lambda(107 \mathrm{~mm} \times 107 \mathrm{~mm})$. The three reflectarrays are composed of $20 \times 20$ unit cells with $\lambda / 2 \times \lambda / 2$ unit cell size. The feeder is a pyramidal horn having aperture dimensions of $(14 \times 12.4 \times 25) \mathrm{mm}$ with WR28 waveguide. This horn is placed at a distance of $10 \lambda$ from the center of the reflectarray such that the F/D ratio is equal to one. The realized horn gain is $12.1 \mathrm{~dB}$ at the frequency of $28 \mathrm{GHz}$ as shown in figure 5. Its $E$ - and $H$-plane half-power beamwidths are $44.3^{\circ}$ and $48^{\circ}$, respectively. Figure 6 shows the phase distribution required on the reflectarray aperture to produce a broadside single beam with center feeding. Figure 7 shows the 3D radiation patterns of the three reflectarrays at $28 \mathrm{GHz}$. The reflectarray with $\mathrm{UC}_{1}$ gives a gain of about $28.5 \mathrm{~dB}$. The reflectarray with $\mathrm{UC}_{2}$ gives a gain of about $28 \mathrm{~dB}$, while the one with $\mathrm{UC}_{3}$ gives a gain of about $25.8 \mathrm{~dB}$. Figure 8 shows a comparison of the $2 \mathrm{D}$ radiation patterns in the $\mathrm{H}$-plane for the designed 

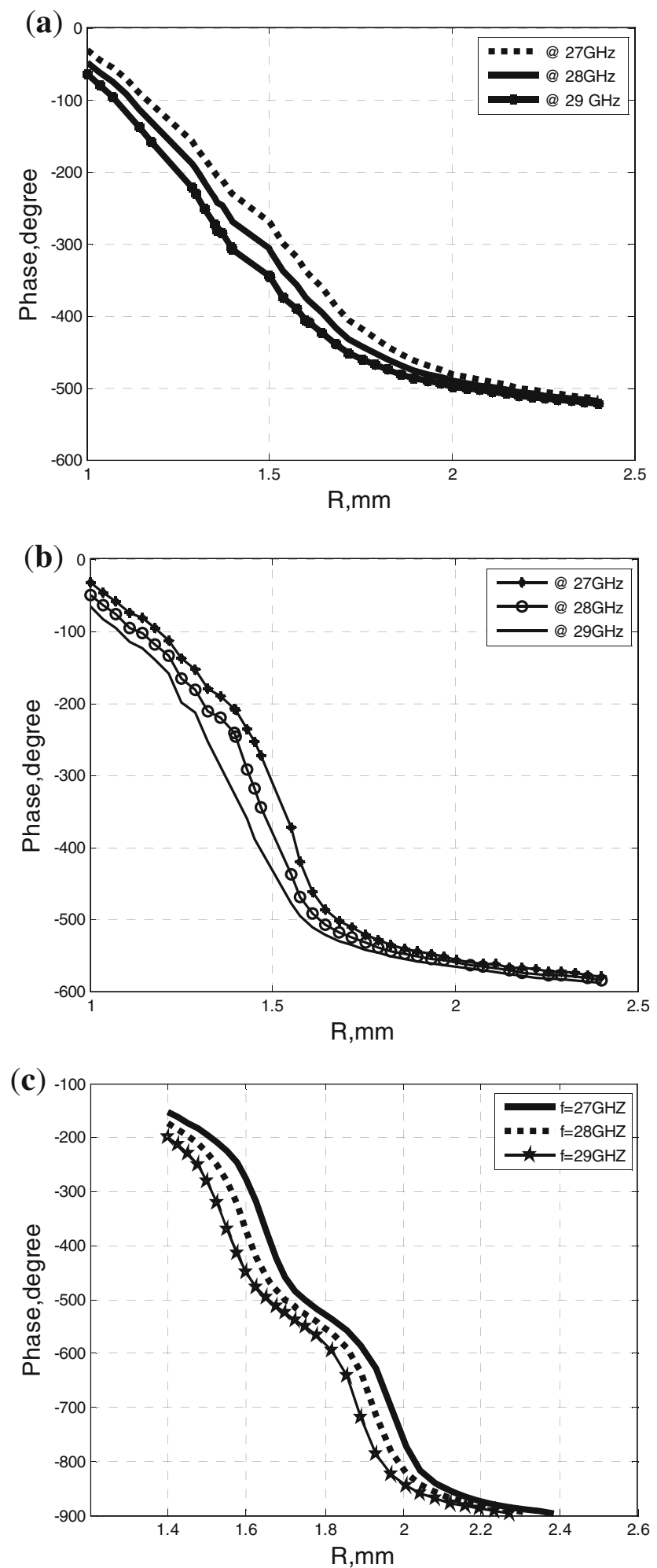

Figure 4. Reflection phase versus $\mathrm{R}$ for (a) $\mathrm{UC}_{1}$, (b) $\mathrm{UC}_{2}$, (c) $\mathrm{UC}_{3}$ with normal incidence only at 27,28 , and $29 \mathrm{GHz}$.

reflectarrays. The far-field gain versus frequency for these reflectarrays is shown in figure 9. From this figure, it is clear that the gain variations with frequency for the

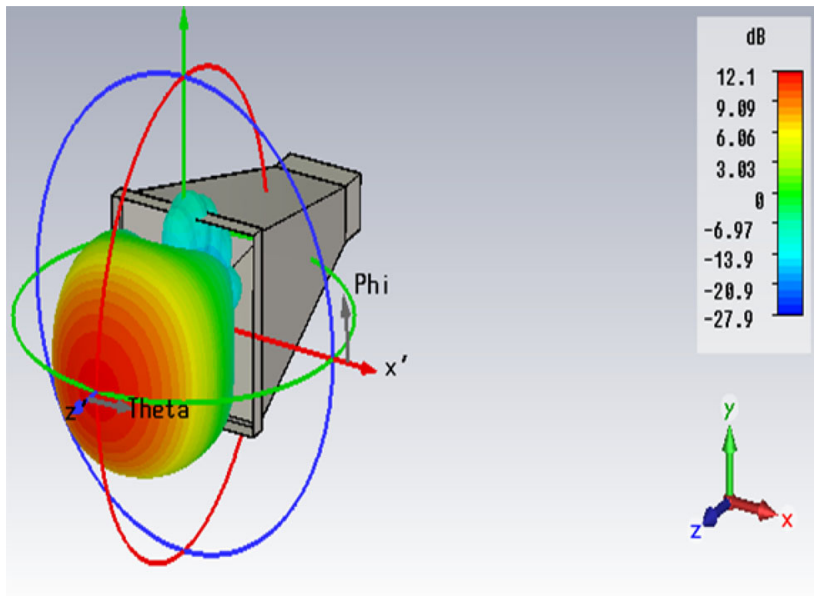

Figure 5. Feeder horn radiation pattern.

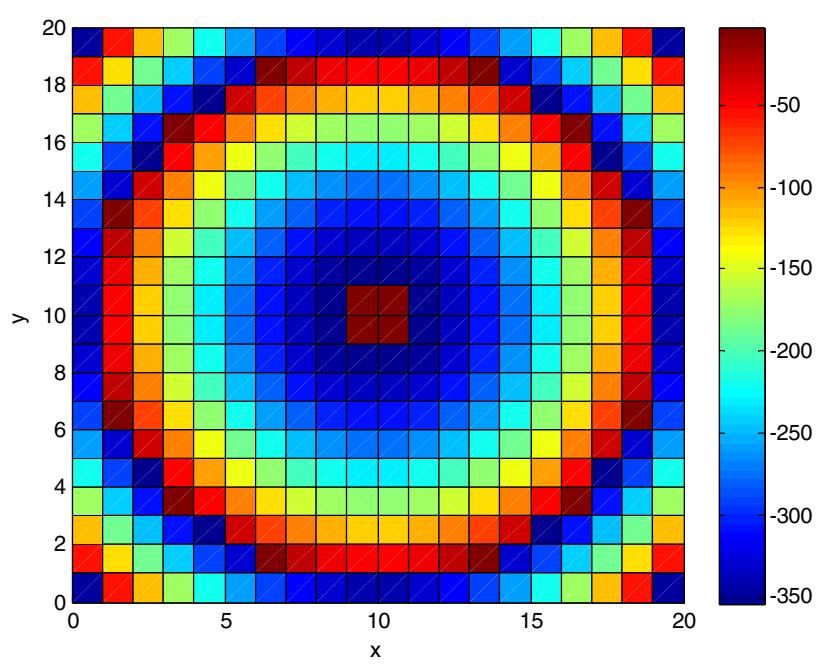

Figure 6. The required phase distribution on the reflectarray surface for the single-beam reflectarray at broadside direction.

reflectarray with $\mathrm{UC}_{1}$ are less than those of the other cases, which means a larger gain-bandwidth value than that of the other cases.

Table 1 presents a comparison between the designed reflectarrays and the reflectarrays in [15] and [16] as they also work in the $\mathrm{Ka}$ band. This table shows that the proposed reflectarray based on $\mathrm{UC}_{1}$ has a gain of about $28.5 \mathrm{~dB}$ and a 1-dB gain-bandwidth equal to $30.7 \%$ despite having a smaller area than those of the reflectarrays in [15] and [16]. The reflectarray with $\mathrm{UC}_{2}$ gives the same results in [15] but with smaller dimensions.

For $\mathrm{UC}_{3}$, another design is presented to produce a single beam at a tilt angle of $10^{\circ}$ from the broadside direction to avoid the blocking that occurs when both the feed and the reflectarray radiations are at the same direction. Figure 10 shows the required phase distribution on the reflectarray 
(a)

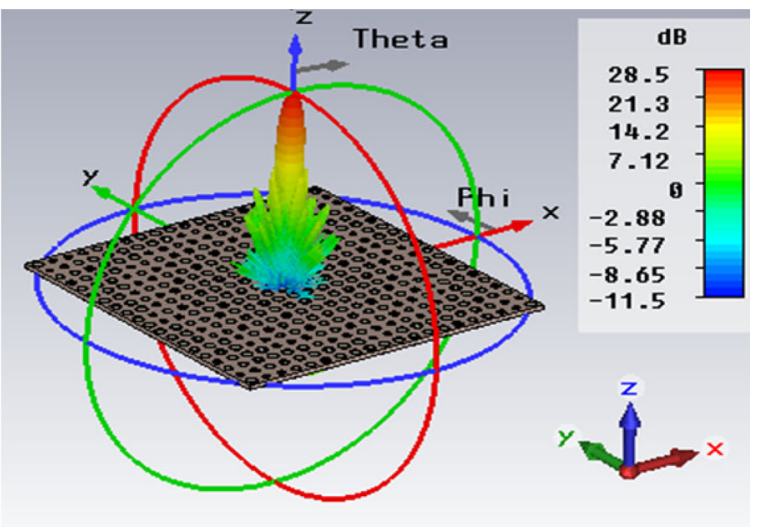

(b)

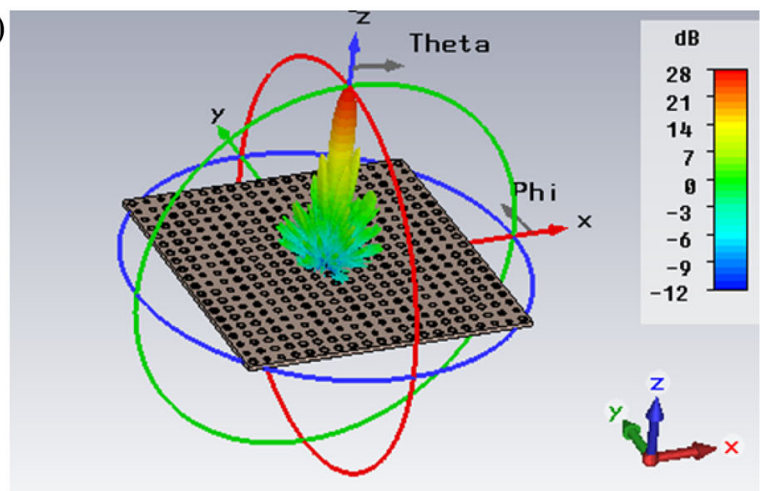

(c)

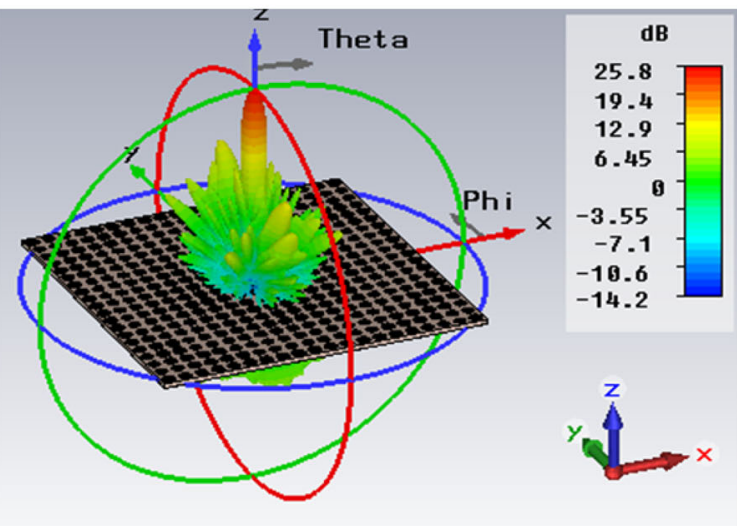

Figure 7. 3D radiation patterns for the reflectarrays at $28 \mathrm{GHz}$ with (a) $\mathrm{UC}_{1}$, (b) $\mathrm{UC}_{2}$, (c) $\mathrm{UC}_{3}$.

surface to collimate a beam at $10^{\circ}$ direction with center feeding. The $2 \mathrm{D}$ radiation patterns for the reflectarray with $\mathrm{UC}_{3}$ for the broadside and $10^{\circ}$ tilt angle are shown in figure 11. The gain is improved from $25.8 \mathrm{~dB}$ at $28 \mathrm{GHz}$ to $26.4 \mathrm{~dB}$, and in addition, the 1-dB gain-bandwidth has been improved from $10.6 \%$ to $19.3 \%$. The SLL has been

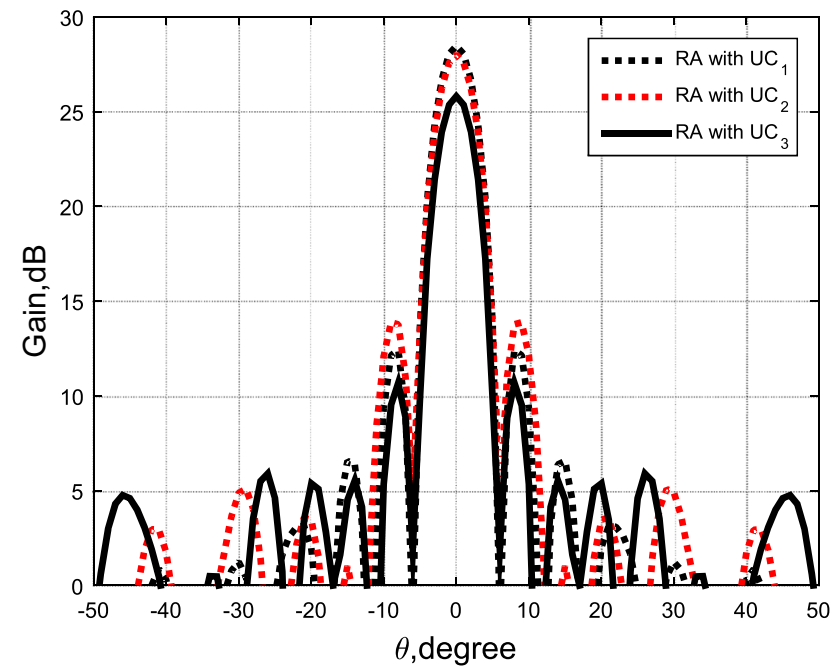

Figure 8. 2D radiation patterns for the proposed reflectarrays at $28 \mathrm{GHz}$.

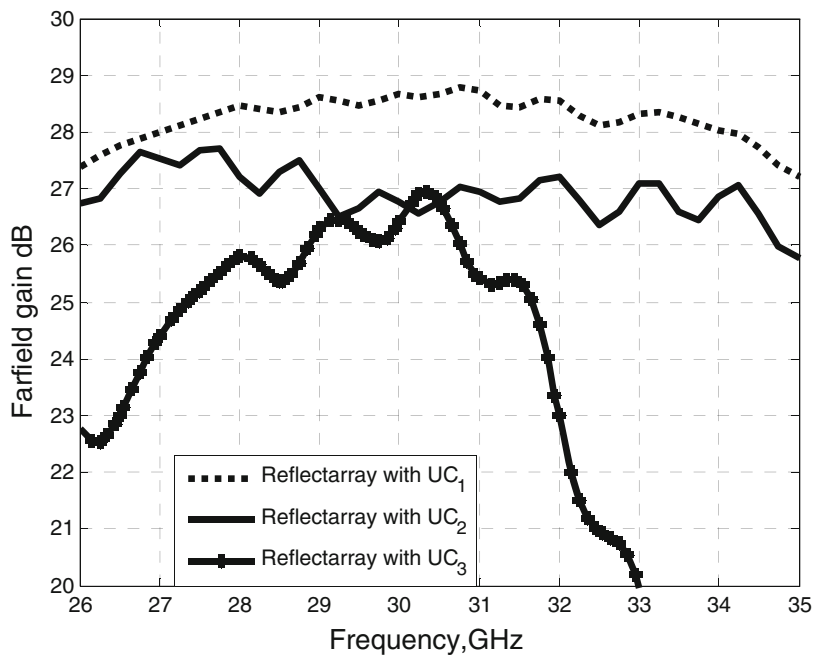

Figure 9. Far-field gain versus frequency for the proposed reflectarrays.

improved from $-13.1 \mathrm{~dB}$ to $-14.7 \mathrm{~dB}$ at the design-frequency. Figure 12 shows the radiation patterns for both cases at different frequencies from $27 \mathrm{GHz}$ to $30 \mathrm{GHz}$. It is clear from this figure that the gain variations for the singlebeam reflectarray with $10^{\circ}$ tilt angle are very small when compared with those of the single-beam reflectarray in the broadside direction with $\mathrm{UC}_{3}$. Table 2 summarizes the results.

\section{Double-beam reflectarrays}

Generally, the objective of designing double-beam antennas is to enhance capacity and spectral efficiency of wireless communication systems by serving multiple users at 
Table 1. Comparison between the proposed reflectarrays and those in [15] and [16].

\begin{tabular}{|c|c|c|c|}
\hline $\begin{array}{l}\text { Reflectarray } \\
\text { with }\end{array}$ & $\begin{array}{c}\text { Aperture } \\
\text { size }(\mathrm{mm})\end{array}$ & $\begin{array}{l}\text { Gain at } \\
\text { designed } \\
\text { frequency } \\
(\mathrm{dB})\end{array}$ & $\begin{array}{l}1 \mathrm{~dB} \text { gain- } \\
\text { bandwidth }\end{array}$ \\
\hline $\mathrm{UC}_{1}$ & $107 \mathrm{~mm} \times 107 \mathrm{~mm}$ & 28.5 & $30.7 \%$ \\
\hline $\mathrm{UC}_{2}$ & $107 \mathrm{~mm} \times 107 \mathrm{~mm}$ & 28 & $10 \%$ \\
\hline $\mathrm{UC}_{3}$ & $107 \mathrm{~mm} \times 107 \mathrm{~mm}$ & 25.8 & $10.6 \%$ \\
\hline $\mathrm{UC}$ in $[15]$ & $120 \mathrm{~mm} \times 120 \mathrm{~mm}$ & 28 & $\cong 10 \%$ \\
\hline $\mathrm{UC}$ in [16] & $160 \mathrm{~mm} \times 160 \mathrm{~mm}$ & 26.3 & $8.75 \%$ \\
\hline
\end{tabular}

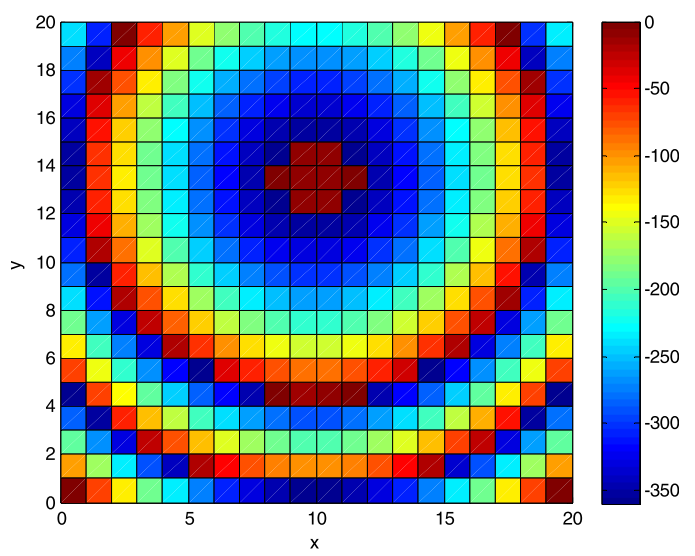

Figure 10. The required phase distribution on the reflectarray surface for the single-beam reflectarray at $10^{\circ}$ tilt angle.

different directions. Usually narrow beams are used for this purpose. In wireless mobile communications, there is a need for an antenna that gives multiple beams. A reflectarray that produces two beams has been designed based on the proposed $\mathrm{UC}_{3}$. There are several methods for designing multi-beam reflectarrays such as superposition of the aperture field method. In this method, to produce $M$ beams using a single feed, the tangential component of the field on the reflectarray surface can be simply written as [12]:

$$
E_{R}\left(x_{i}, y_{j}\right)=\sum_{1}^{M} A_{n, i}\left(x_{i} \cdot y_{j}\right) e^{j \phi_{n, i}\left(x_{i}, y_{i}\right)}
$$

where $A_{n, i}$ and $\phi_{n, i}$ are the amplitude and phase of each element to radiate a beam in the direction $\left(\theta_{n}, \phi_{n}\right)$. In reflectarrays, the amplitude of each element is fixed by the feed position and element location, which are independent of the beam direction. Therefore [12]:

$$
E_{R}\left(x_{i}, y_{j}\right)=A_{i}^{f e e d}\left(x_{i}, y_{j}\right) \sum_{1}^{M} e^{j \phi_{n, i}\left(x_{i}, y_{i}\right)}
$$

Generally, in the reflectarray design with the superposition method, it is required to achieve certain magnitude and phase at each element of the reflectarray. It is possible to achieve the phase requirements, but the magnitude

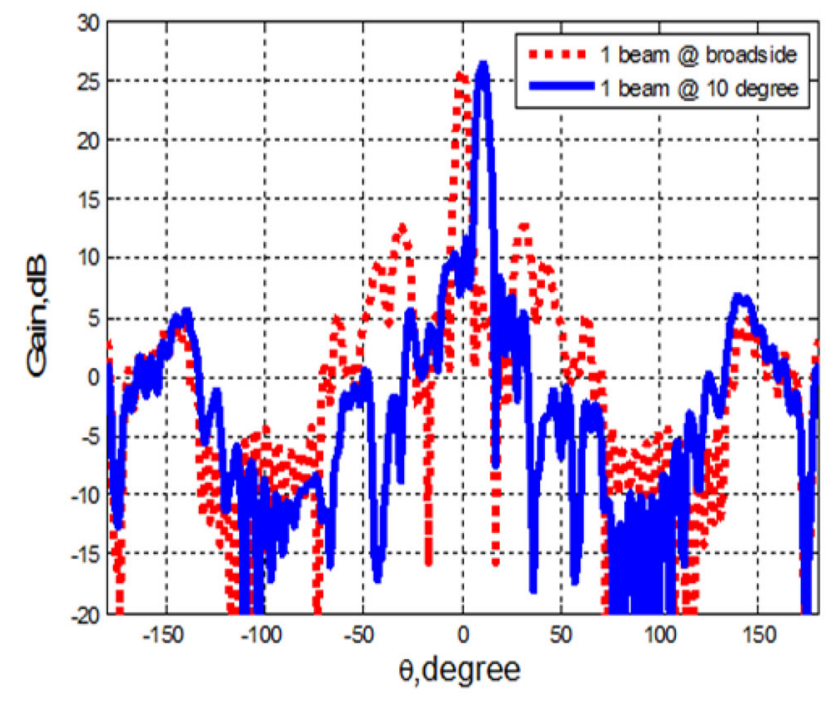

Figure 11. 2D radiation pattern for the single-beam reflectarray with $\mathrm{UC}_{3}$.
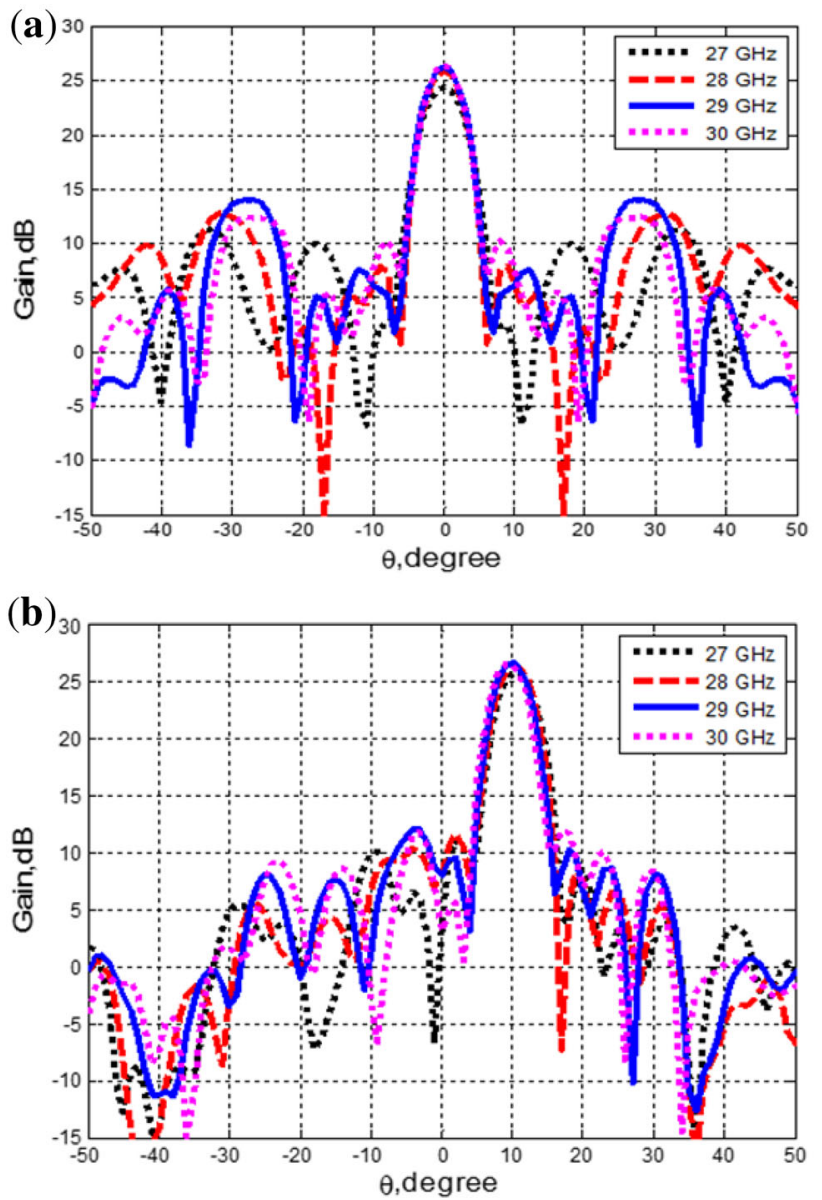

Figure 12. $2 \mathrm{D}$ radiation pattern for the single-beam reflectarray using $\mathrm{UC}_{3}$ at $27 \mathrm{GHz}, 28 \mathrm{GHz}, 29 \mathrm{GHz}$, and $30 \mathrm{GHz}$ (a) broadside direction, (b) $10^{\circ}$ tilt angle. 
Table 2. Characteristics of the reflectarray with $\mathrm{UC}_{3}$.

\begin{tabular}{|c|c|c|c|c|c|c|c|}
\hline \multirow[b]{2}{*}{$\begin{array}{l}\text { Single-beam reflectarrays } \\
\text { with a beam at }\end{array}$} & \multicolumn{4}{|c|}{ Gain $(\mathrm{dB})$} & \multirow[b]{2}{*}{$1 \mathrm{~dB}$ Gain-bandwidth } & \multirow[b]{2}{*}{$\begin{array}{l}\text { SLL @ } 28 \\
\mathrm{GHz}(\mathrm{dB})\end{array}$} & \multirow[b]{2}{*}{$\begin{array}{c}\text { Beamwidth@28 } \\
\text { GHz }\end{array}$} \\
\hline & $\begin{array}{l}\text { @ } 27 \\
\mathrm{GHz}\end{array}$ & $\begin{array}{l}@ 28 \\
\mathrm{GHz}\end{array}$ & $\begin{array}{l}@ 29 \\
\mathrm{GHz}\end{array}$ & $\begin{array}{l}@ 30 \\
\mathrm{GHz}\end{array}$ & & & \\
\hline Broadside direction & 24.4 & 25.8 & 26.3 & 26.4 & $(30.182-27.21) \cong 10.6 \%$ & -13.1 & $5.2^{\circ}$ \\
\hline $10^{\circ}$ & 25.8 & 26.4 & 26.6 & 26.6 & $(32.076-26.593) \cong 19.3 \%$ & -14.7 & $5.4^{\circ}$ \\
\hline
\end{tabular}

requirements are not attainable with the phase synthesis method. This approach has some shortcomings such as the high SLL and gain reduction due to amplitude error. Another possible design approach is the geometrical approach. In this approach, the reflectarray surface is divided into $M$ sub-arrays, and then each sub-array is designed independently to radiate a beam in a desired direction. In this paper, four double-beam reflectarrays will be introduced depending on the geometrical design approach. The two beams are designed at different angles of $5^{\circ}, 10^{\circ}, 15^{\circ}$, and $20^{\circ}$ by dividing the whole array into two sub-arrays such that each sub-array produces one beam with $\theta=5^{\circ}, 10^{\circ}, 15^{\circ}$, or $20^{\circ}$, and $\phi_{1,2}=0^{\circ}, 180^{\circ}$. The phase distributions required on the reflectarray surfaces for the four antennas are shown in figure 13. These reflectarrays are simulated and compared. (a)

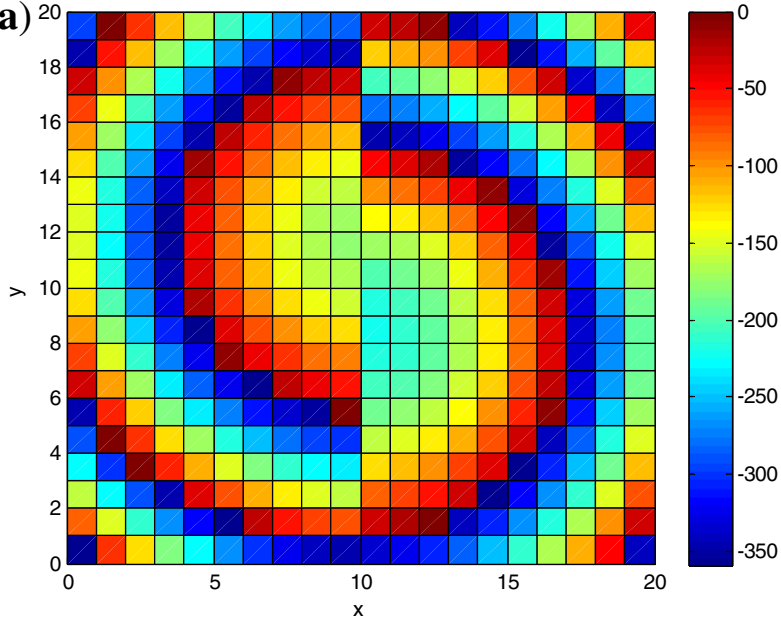

(b)

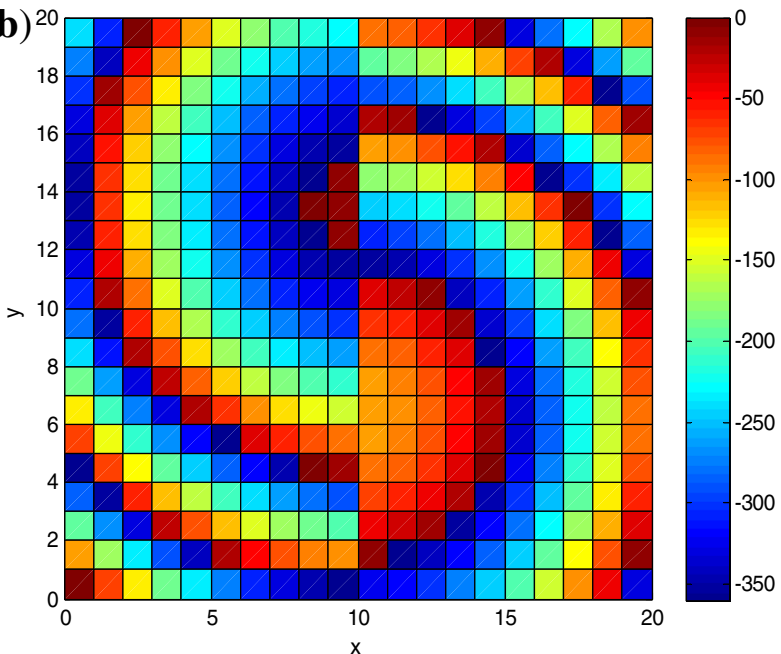

(c)

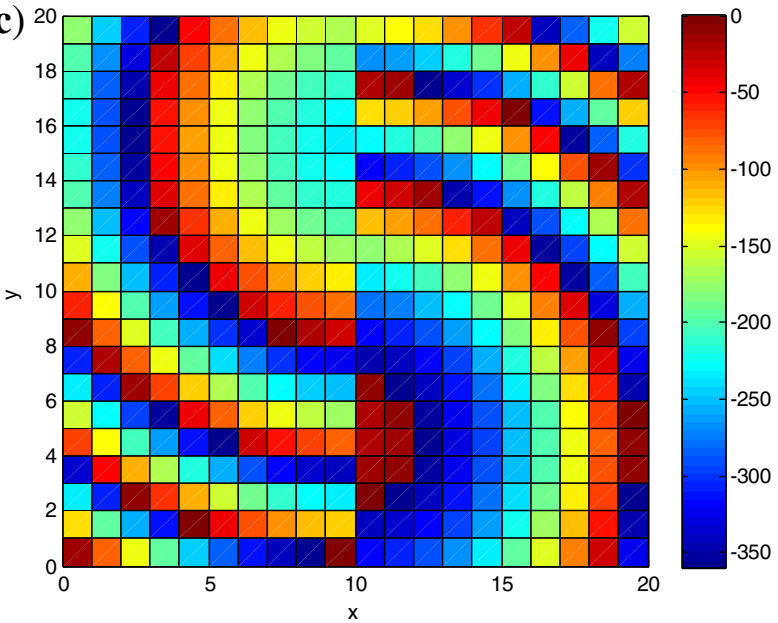

(d)

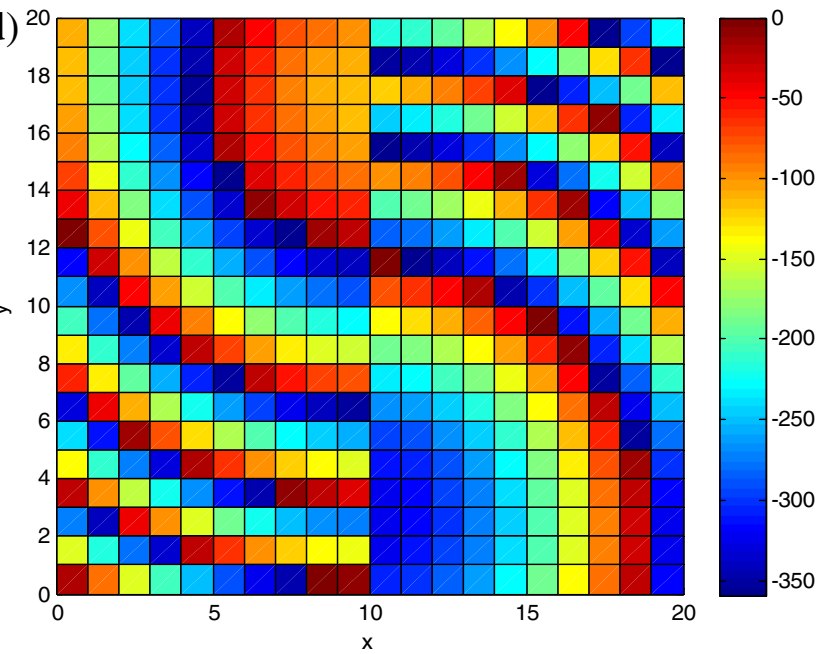

Figure 13. The required phase distributions for the double-beam reflectarrays at (a) $\theta=5^{\circ}$, (b) $\theta=10^{\circ},(\mathbf{c}) \theta=15^{\circ}$ and (d) $\theta=20^{\circ}$ and $\phi_{1,2}=0^{\circ}$ and $180^{\circ}$. 
(a)

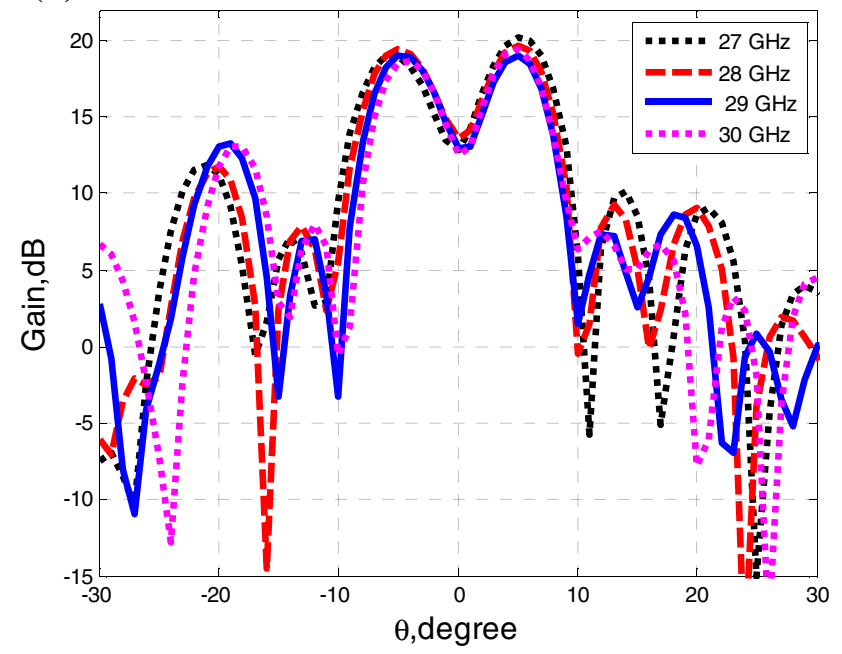

(b) Far-field gain 2 beams @ 10 deg

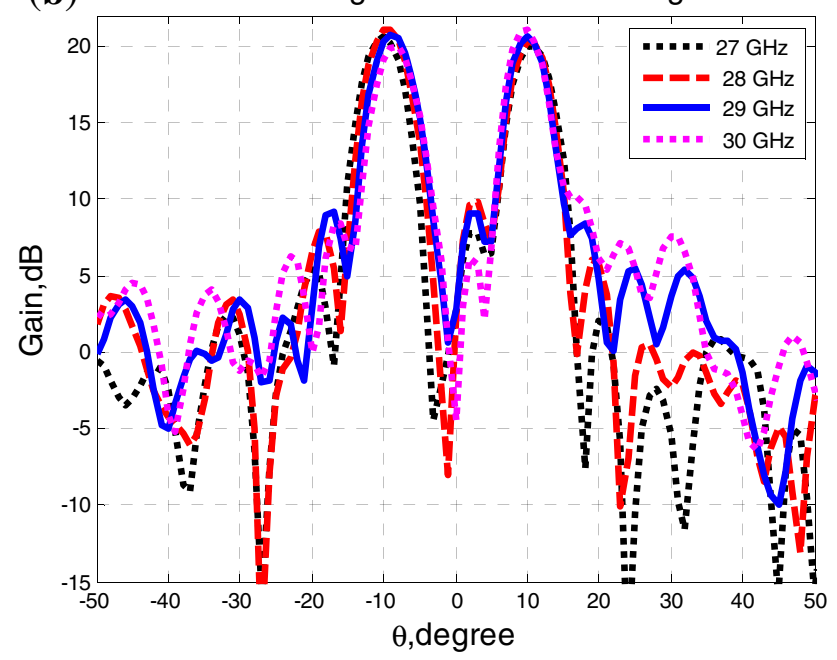

(c) Far-field gain 2 beams @ 15 deg

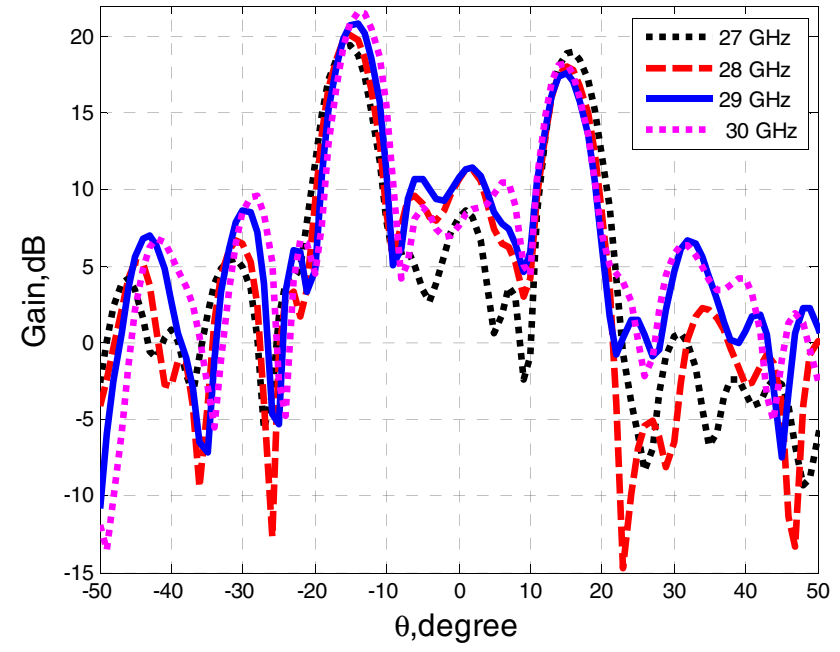

(d)

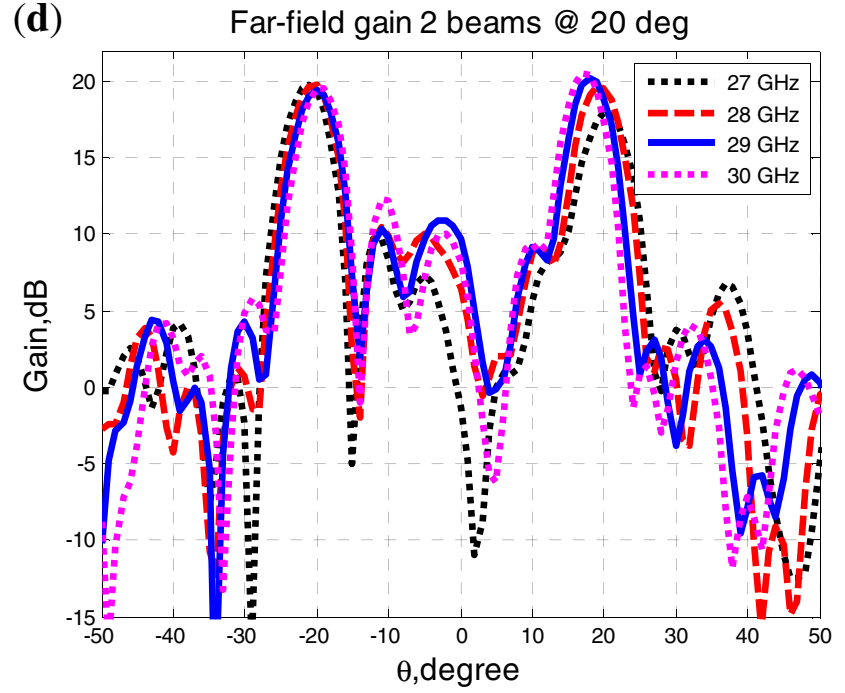

Figure 14. 2D radiation pattern for the double-beam reflectarrays at $27 \mathrm{GHz}, 28 \mathrm{GHz}, 29 \mathrm{GHz}$, and $30 \mathrm{GHz}$ at (a) $5^{\circ}$ (b) $10^{\circ}$ (c) $15^{\circ}$ (d) $20^{\circ}$.

\section{Simulation results}

For the double-beam scenario, a study is presented for the reflectarray designs for two beams with tilt angles of $5^{\circ}$, $10^{\circ}, 15^{\circ}$, and $20^{\circ}$. Figure 14 illustrates the $2 \mathrm{D}$ radiation patterns for the double-beam reflectarrays using the proposed $\mathrm{UC}_{3}$ at different angles in the range from $27 \mathrm{GHz}$ to $30 \mathrm{GHz}$. It is clear from this figure that the best case of distinguishable beams is that for the reflectarray with two beams at a tilt angle of $10^{\circ}$, because at this angle the side

Table 3. Characteristics of the double-beam reflectarrays.

\begin{tabular}{|c|c|c|c|c|c|c|c|c|c|c|}
\hline \multirow[b]{3}{*}{$\begin{array}{l}\text { Double-beam reflectarrays with } \\
\text { tilt angle at }\end{array}$} & \multicolumn{8}{|c|}{ Gain (dB) } & \multirow[b]{3}{*}{ Beamwidth } & \multirow[b]{3}{*}{$\begin{array}{l}\text { SLL@ } 28 \mathrm{GHz} \\
(\mathrm{dB})\end{array}$} \\
\hline & \multicolumn{2}{|c|}{ @ 27 GHz } & \multicolumn{2}{|c|}{ @ $28 \mathrm{GHz}$} & \multicolumn{2}{|c|}{ @ $29 \mathrm{GHz}$} & \multicolumn{2}{|c|}{ (a) $30 \mathrm{GHz}$} & & \\
\hline & $\begin{array}{c}1^{\text {st }} \\
\text { beam }\end{array}$ & $\begin{array}{c}2^{\text {nd }} \\
\text { beam }\end{array}$ & $\begin{array}{c}1^{\text {st }} \\
\text { beam }\end{array}$ & $\begin{array}{c}2^{\text {nd }} \\
\text { beam }\end{array}$ & $\begin{array}{c}1^{\text {st }} \\
\text { beam }\end{array}$ & $\begin{array}{c}2^{\text {nd }} \\
\text { beam }\end{array}$ & $\begin{array}{c}1^{\text {st }} \\
\text { beam }\end{array}$ & $\begin{array}{l}2^{\text {nd }} \\
\text { beam }\end{array}$ & & \\
\hline $5^{\circ}$ & 19 & 20.2 & 19.4 & 19.7 & 19 & 19.1 & 18.7 & 19.4 & $4.9^{\circ}$ & -7.9 \\
\hline $10^{\circ}$ & 20.6 & 19.8 & 21.1 & 20.2 & 20.8 & 20.7 & 20 & 21.1 & $5.7^{\circ}$ & -11.2 \\
\hline $15^{\circ}$ & 19 & 19.2 & 20.1 & 18 & 20.9 & 17.5 & 21.6 & 18.2 & $5.3^{\circ}$ & -8.7 \\
\hline $20^{\circ}$ & 19.7 & 17.8 & 19.7 & 19.7 & 19.3 & 20.2 & 19.5 & 20.6 & $5.4^{\circ}$ & -9.3 \\
\hline
\end{tabular}




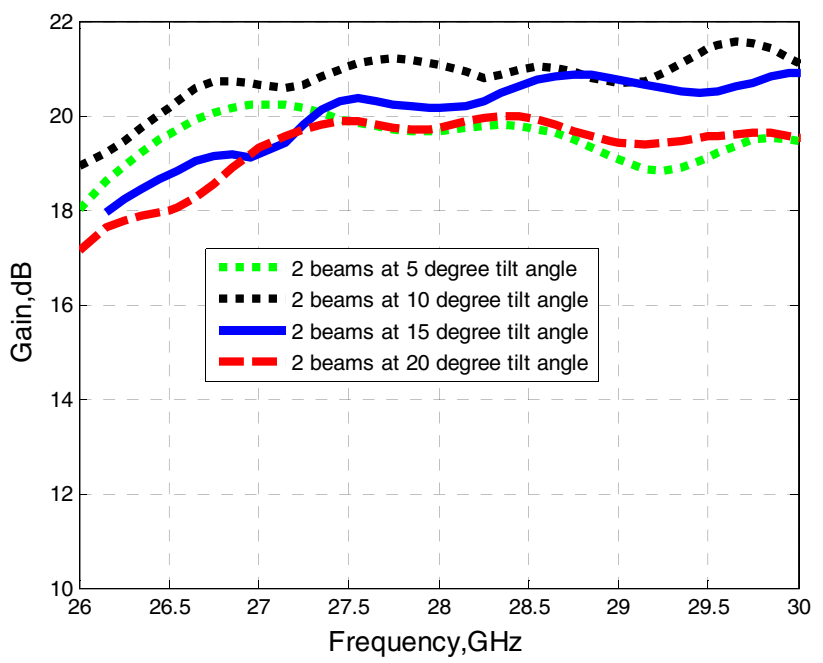

Figure 15. Far-field gain versus frequency for the double-beam reflectarrays.

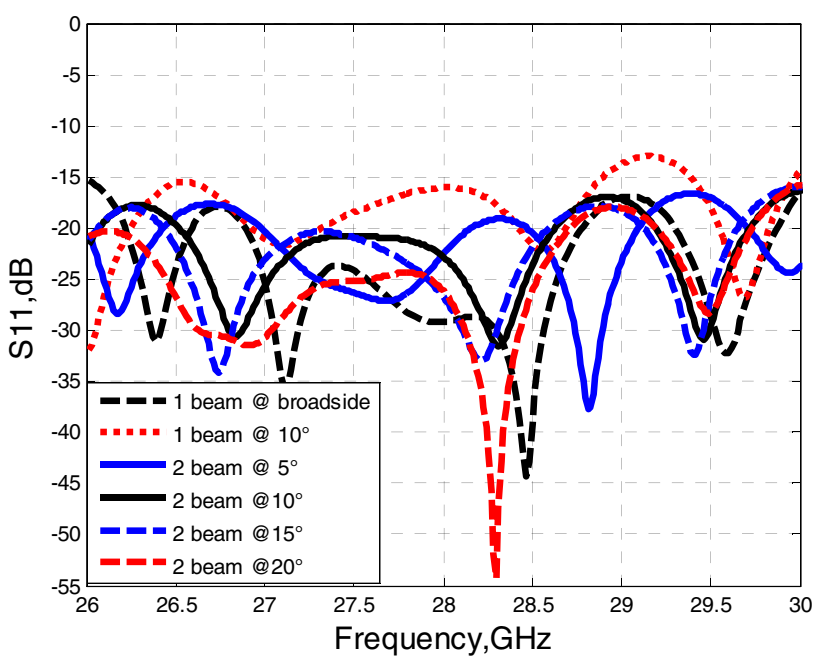

Figure 16. Reflection coefficient for all reflectarrays

lobes overlap is minimal. The values of the gain are given in Table 3 in detail. From this table, it is clear that the double-beam reflectarray with $10^{\circ}$ tilt angle is the best from the gain and SLL perspectives in addition to the $1 \mathrm{~dB}$ gainbandwidth value. The gain variation in this case is small when compared with the other cases.

Figure 15 shows the far-field gain versus frequency for the double-beam cases. The gain variation over the frequency range of interest, for the all cases, is less than $1 \mathrm{~dB}$. This means a high 1-dB gain-bandwidth, which is verified through the reflection coefficient figure. The reflection coefficient for all reflectarrays is shown in figure 16. From this figure, it is clear that the matching condition is guaranteed over the operating frequency range for the designed reflectarrays. (a)
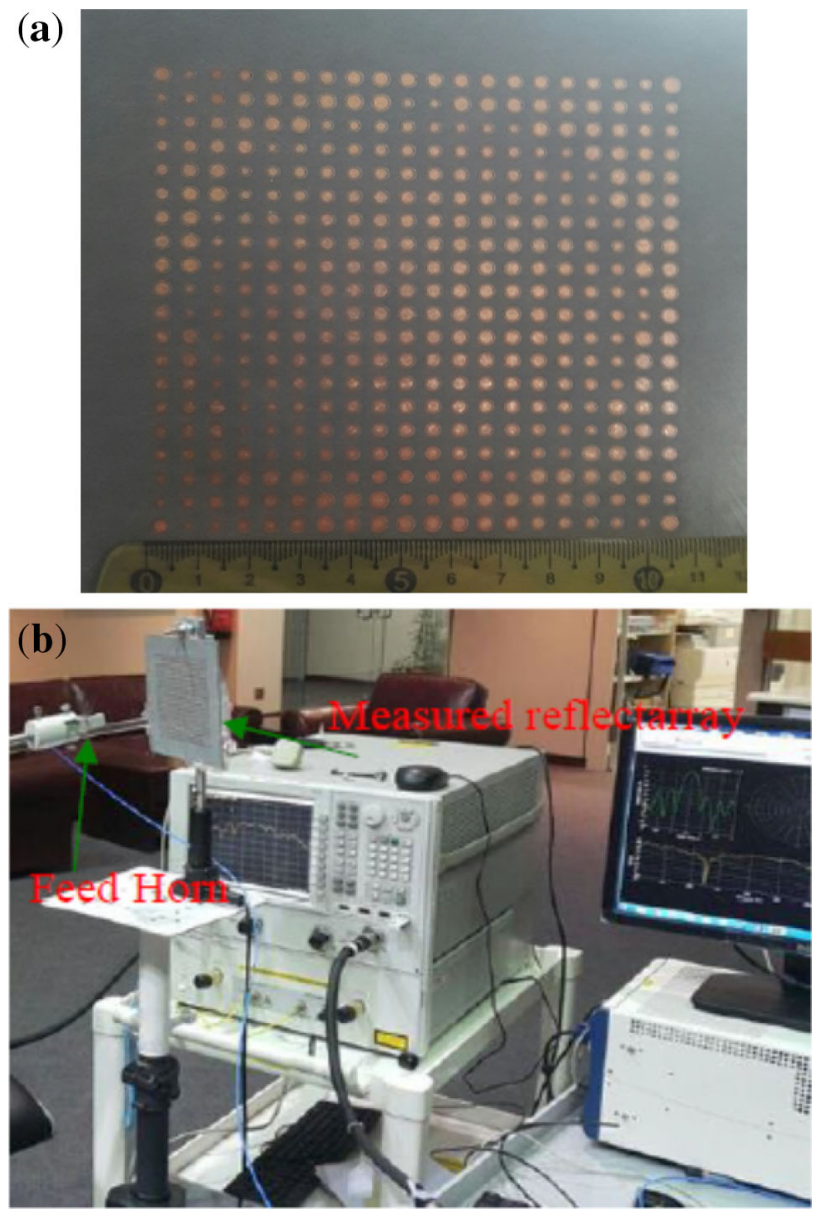

Figure 17. (a) Photo of the fabricated reflectarray with $\mathrm{UC}_{1}$, (b) reflectarray with $\mathrm{UC}_{1}$ under test.

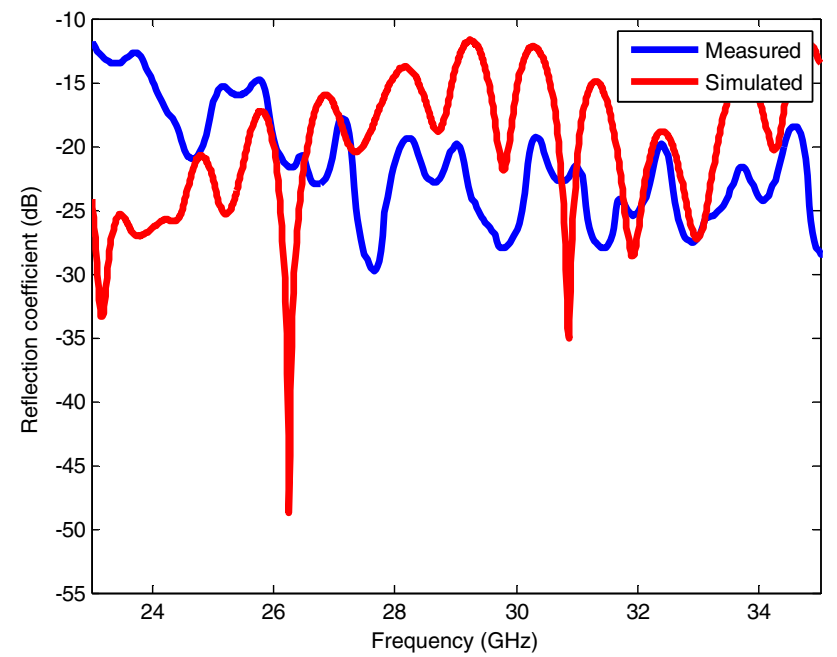

Figure 18. Reflection coefficient versus frequency for the simulation and measurements of the reflectarray with $\mathrm{UC}_{1}$. 


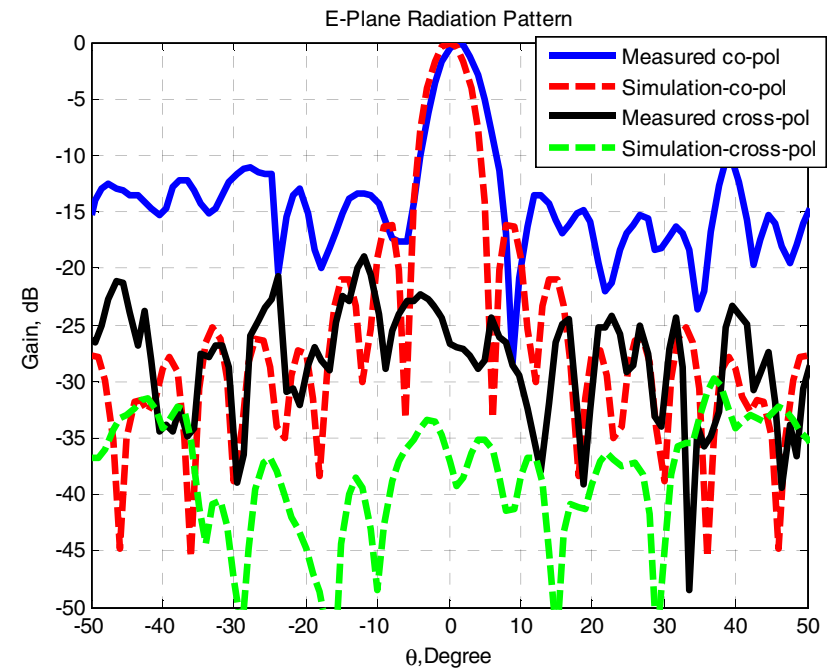

Figure 19. E-plane radiation pattern comparison between the simulated and fabricated reflectarrays with $\mathrm{UC}_{1}$.

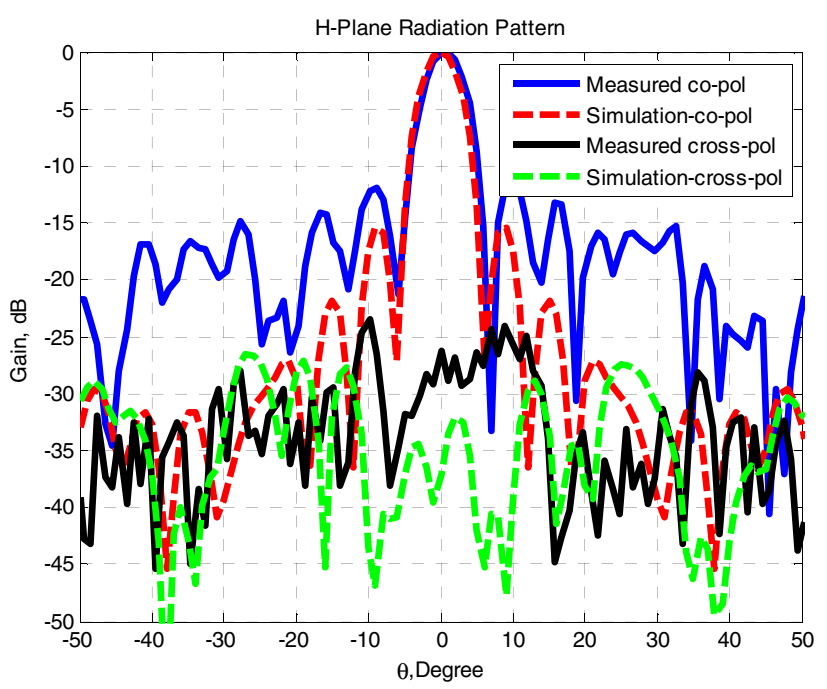

Figure 20. H-plane radiation pattern comparison between the simulated and fabricated reflectarrays with $\mathrm{UC}_{1}$.

\section{Fabrication and measurements}

Two reflectarrays with dimensions $107 \mathrm{~mm} \times 107 \mathrm{~mm}$ with unit cell dimensions of $5.35 \mathrm{~mm} \times 5.35 \mathrm{~mm}$ have been fabricated and measured. These reflectarrays have been fed with a pyramidal horn placed at the center of the array at a distance of $107 \mathrm{~mm}$ from the array surface. The antenna gives one beam at the broadside direction. Figure 17 (a) shows a photo of the fabricated reflectarray with $\mathrm{UC}_{1}$. Figure $17(\mathrm{~b})$ shows the fabricated reflectarray under test. Figure 18 shows a comparison between the simulated and the measured reflection coefficient values for the fabricated reflectarray. Figures 19 and 20 show a good

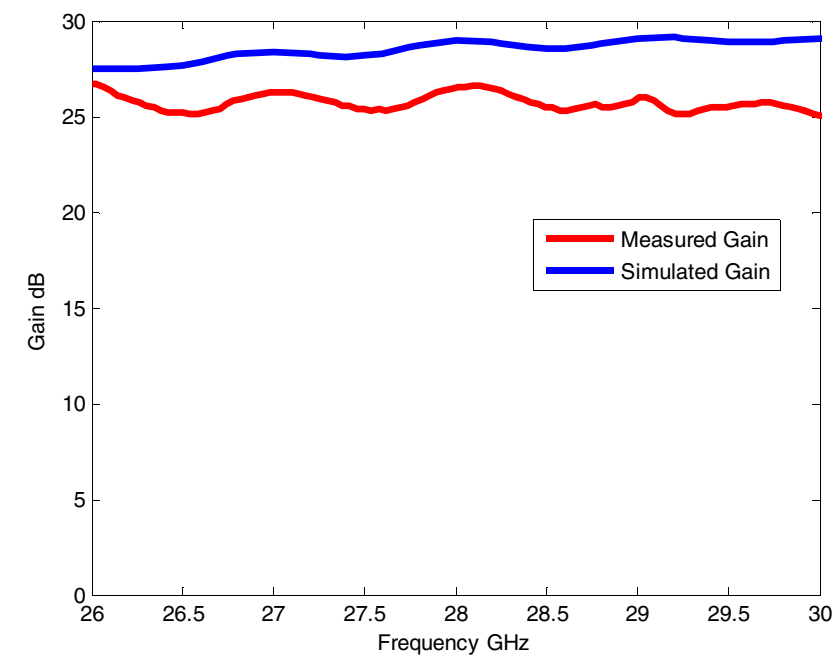

Figure 21. Comparison between the gains of the simulated and fabricated reflectarrays with $\mathrm{UC}_{1}$ versus frequency.

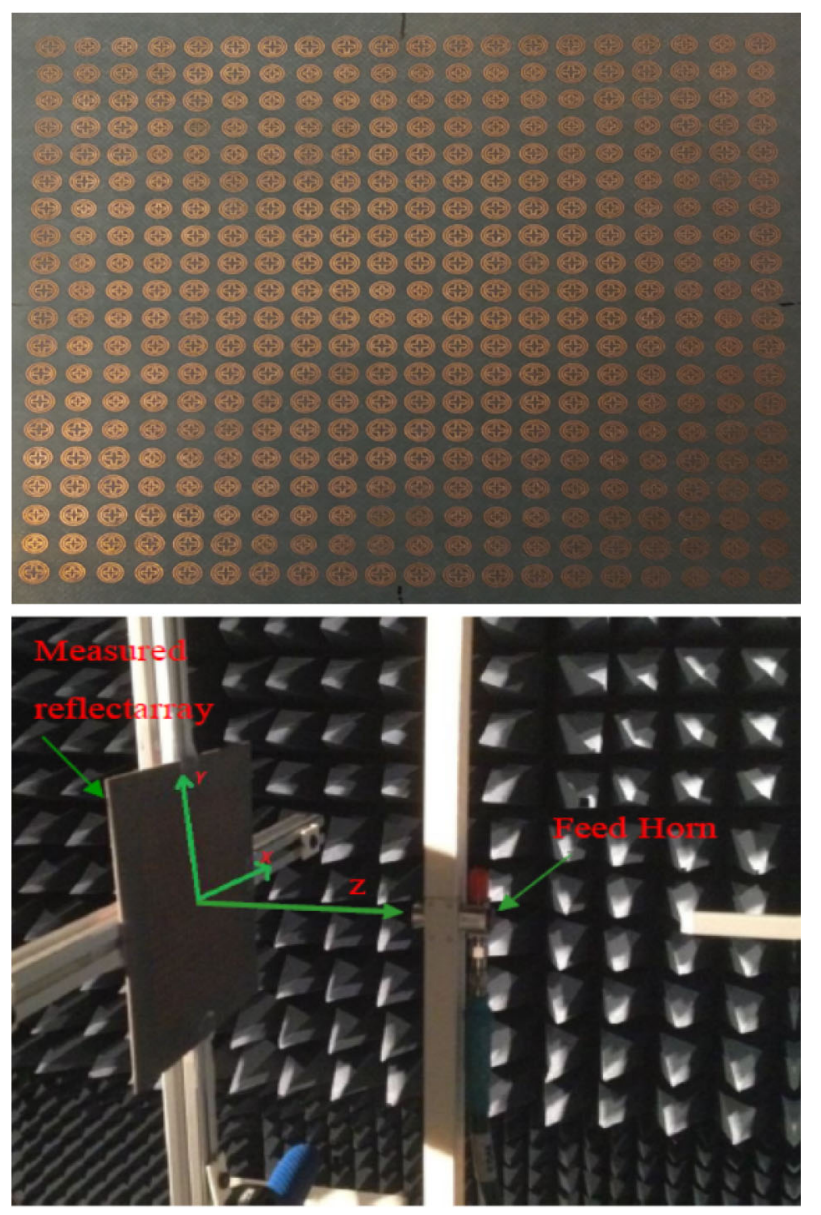

Figure 22. (a) Photo of the fabricated reflectarray with $\mathrm{UC}_{3-}$, (b) reflectarray with $\mathrm{UC}_{3}$ under test.

agreement between the measured and simulated radiation patterns for both E- and H-planes. Figure 21 shows a comparison between the measured and simulated gain over 


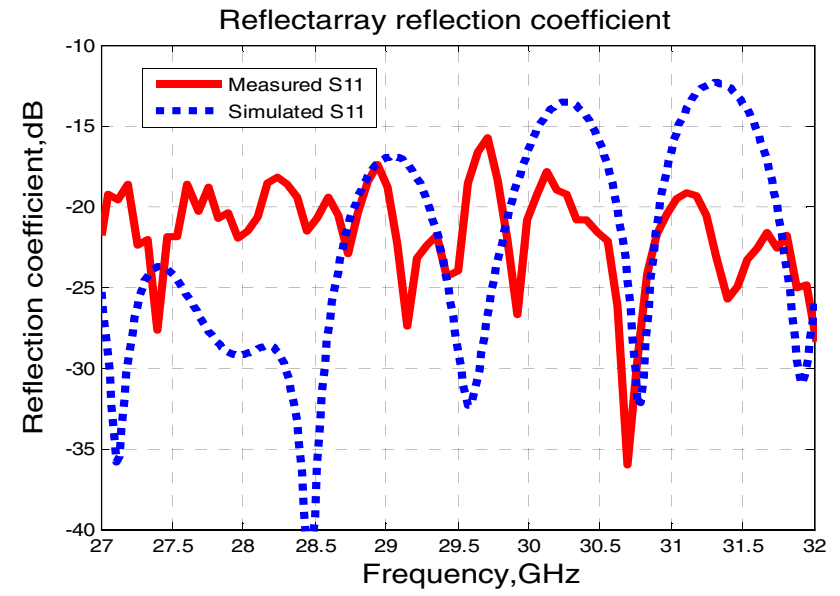

Figure 23. Reflection coefficient comparison between the simulated and fabricated reflectarrays with $\mathrm{UC}_{3}$.

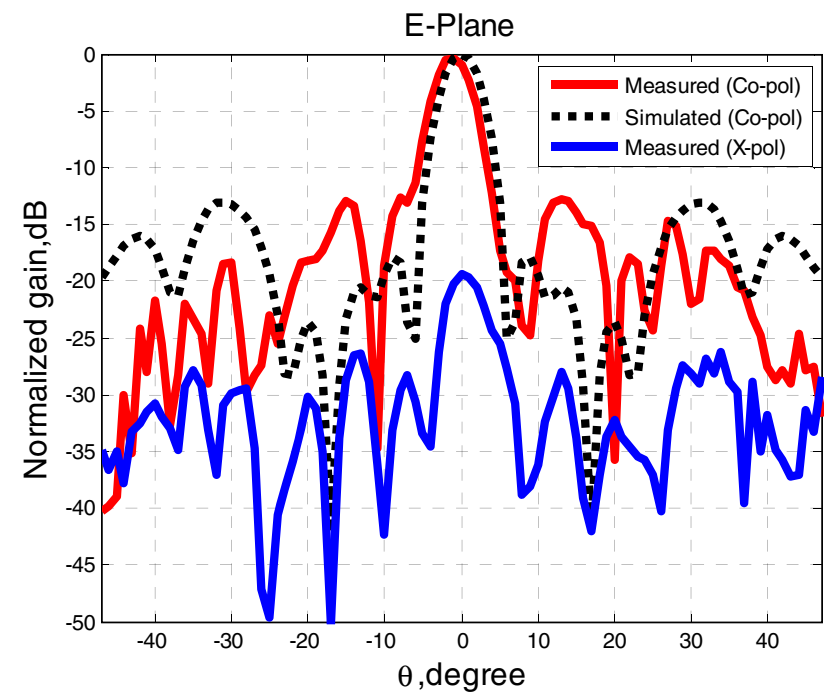

Figure 24. E-plane radiation pattern comparison between the simulated and fabricated reflectarrays with $\mathrm{UC}_{3}$.

the frequency band from 27 to $32 \mathrm{GHz}$. The difference between the simulated and measured results is due to misalignment in the measurement set-up, fabrication errors and material losses. The simulation results confirm gain flatness of the fabricated reflectarray over the frequency band of interest. Figures 22-25 reveal another measurement experiment with its results for the reflectarray designed based on $\mathrm{UC}_{3}$. This experiment illustrates also the gain flatness over the frequency band of interest and the good match between simulation and measurement results (figure 26).

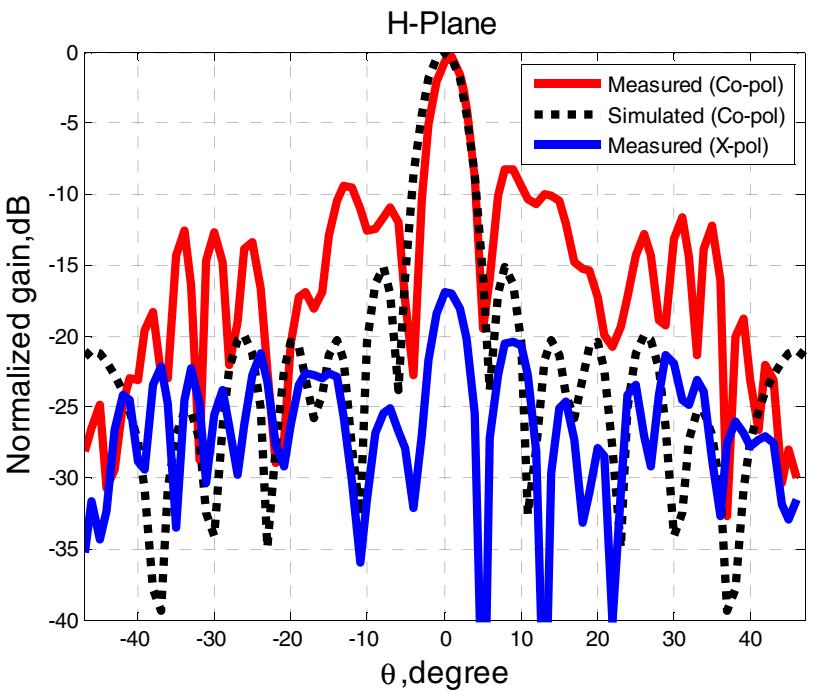

Figure 25. H-plane radiation pattern comparison between the simulated and fabricated reflectarrays with $\mathrm{UC}_{3}$.

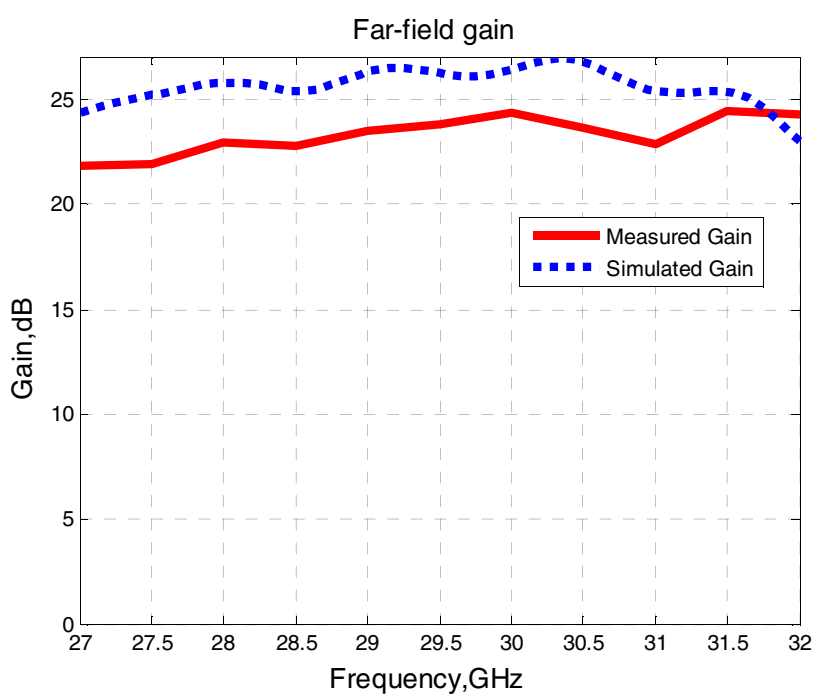

Figure 26. Comparison between the gains of the simulated and fabricated reflectarrays with $\mathrm{UC}_{3}$ versus frequency.

\section{Conclusion}

Different designs for single- and double-beam reflectarrays suitable for millimeter wave communication at $28 \mathrm{GHz}$ have been presented in this study. These designs can be efficiently used in the sectored mobile cells recommended for future generation mobile communication systems. Simulation results revealed the high gain-bandwidth of the designed reflectarrays. Moreover, two prototypes for a single-beam reflectarray have been fabricated, and their radiation characteristics have been measured. The measured and simulated results are in good match revealing 
good gain-bandwidth characteristics over the desired frequency band.

\section{List of symbols}

$\lambda_{o}$ free space wavelength

$N$ integer number

$f_{o} \quad$ center frequency

$D$ the reflectarray aperture diameter

$F \quad$ focal length

$k_{o}$ free-space wavenumber at the design frequency

$c$ speed of light

$\gamma_{i}$ the $i$ th element phase required to compensate for the delay resulting from the corresponding path

\section{References}

[1] Agiwal M, Roy A and Saxena N 2016 Next generation 5G wireless networks: a comprehensive survey. IEEE Commun. Surv. Tutor. 18: 1617-1655

[2] Huang K and Edwards D J 2008 Millimetre wave antennas for gigabit wireless communications. Wiley, Hoboken

[3] Tagonski S D and Pozar D M 1994 Analysis and design of a microstrip reflectarray using patches of variable size. In: Proceedings of IEEE Antennas and Propagation Society International Symposium and URSI National Radio Science Meeting, vol. 3, pp. 1820-1823

[4] Huang J and Encinar J A 2008 Reflectarray antennas. Wiley, Hoboken

[5] Guha D and Antar Y 2011 Microstrip and printed antennas new trends, techniques and applications. Wiley, Hoboken

[6] Nayeri P, Yang F and Elsherbeni A Z 2013 Design of singlefeed reflectarray antennas with asymmetric multiple beams using the particle swarm optimization method. IEEE Trans. Antennas Propag. 61: 4598-4605

[7] Nayeri P, Yang F and Elsherbeni A Z 2012 Design and experiment of a single-feed quad-beam reflectarray antenna. IEEE Trans. Antennas Propag. 60:1166-1171

[8] Fang X, Wu Q and Yang G 2014 Dual-beam realization of reflectarray antenna based on phase synthesis method (PSM). In: Proceedings of 3rd Asia-Pacific on Antennas and Propagation, pp. 603-606

[9] Arrebola M, Encinar J A and Barba M 2008 Multifed printed reflectarray with three simultaneous shaped beams for LMDS central station antenna. IEEE Trans. Antennas Propag. 56: $1518-1527$

[10] Tyapkin V N, Kartsan I N, Dmitriev D D and Goncharov A E 2015 Spatial filtering algorithms in adaptive multi-beam hybrid reflector antennas. In: Proceedings of International Siberian on Control and Communications (SIBCON), pp. 1-5

[11] Qu Y, Guo C, Guo H and Ding J 2015 Design of single-feed multi-beam reflectarray using iterative fourier techniques. Int. J. Smart Home 9: 187-194

[12] Martinez-de-Rioja E, Encinar J A, Florencio R and Boix R R 2016 Reflectarray in $\mathrm{K}$ and $\mathrm{Ka}$ bands with independent beams in each polarization. In: Proceedings of the AP-S, pp. $1199-1200$

[13] Dai X and Zhou T 2016 Dual-band reflectarray with crosseddipole elements for GSM and LTE applications. AEU Int. J. Electron. Commun. 70: 605-610

[14] Mahmoud A, Kishk A A, Hao Z and Hong W 2016 Ka-band circularly polarized reflectarray using a double-layers cross slot. IEEE Antennas Propag. Mag. 58: 60-68

[15] [15] Zhang S 2017 Three-dimensional printed millimetre wave dielectric resonator reflectarray. IET Microw. Antennas Propag. 11: 2005-2009

[16] An W, Xu S, Yang F and Gao J 2014 A Ka-band reflectarray antenna integrated with solar cells. IEEE Trans. Antennas Propag. 62: 5539-5546

[17] Hong W, Jiang Z H, Yu C, Zhou J, Chen P, Yu Z, Zhang H, Yang B, Pang X, Jiang M, Cheng Y, Al-Nuaimi M K T, Zhang Y, Chen J and He S 2017 Multibeam antenna technologies for $5 \mathrm{G}$ wireless communications. IEEE Trans. Antennas Propag. 65: 6231-6249

[18] Dahri M H, Jamaluddin M H, Abbasi M I and Kamarudin M R 2017 A review of wideband reflectarray antennas for $5 \mathrm{G}$ communication systems. IEEE Access 5: 17803-17815

[19] Chaharmir M R and Shaker J 2008 Broadband reflectarray with combination of cross and rectangle loop elements. Electron. Lett. 44: 658-659

[20] Chaharmir M R, Shaker J and Gagnon N 2009 Broadband dual-band linear orthogonal polarisation reflectarray. Electron. Lett. 45: 13-14

[21] [21] Misran N, Cahill R and Fusco V F 2003 Design optimization of ring elements for broadband reflectarray antennas. IEE Proc. Antennas Propag. 150: 440-444

[22] Bialkowski M E and Sayidmarie K H 2008 Investigations into phase characteristics of a single-layer reflectarray employing patch or ring elements of variable size. IEEE Trans. Antennas Propag. 56: 3366-3372

[23] Elsharkawy R, Sebak A -R, Hindy M, Haraz O M, Saleeb A and El-Rabaie S M 2015 Polarization insensitive Ka-band reflectarray antenna. In: Proceedings of the AP-S, pp. 2483-2484

[24] Elsharkawy R, Sebak A -R, Hindy M, Haraz O M, Saleeb A and El-Rabaie S M 2015 Single layer polarization independent reflectarray antenna for future $5 \mathrm{G}$ cellular applications. In: International Conference on Information and Communication Technology Research, pp. 9-12

[25] CST-Computer Simulation Technology. Documentation. www.cst.com 